\title{
LA SUSPENSIÓN DEL ACTO RECLAMADO A “CONTRARIO SENSU” DEL JUICIO DE AMPARO
}

\author{
THE SUSPENSION OF THE ACT CLAIMED TO “CONTRARIO SENSU” OF THE \\ AMPARO TRIAL
}

\begin{abstract}
José Refugio Rosiles-Sánchez ${ }^{1}$
1. Escuela Judicial, Centro de Especialización Judicial. Poder Judicial del Estado de Tabasco, México. rosilesjr@gmail.com
\end{abstract}

* Autor de correspondencia: José Refugio Rosiles-Sánchez, correo electrónico: rosilesjr@gmail.com

\section{RESUMEN}

Este documento expone la suspensión del acto reclamado como la Institución Procesal que es, su importancia y trascendencia, cuya finalidad es mantener viva la materia que da origen al Juicio de Amparo, congelando el actuar de la Autoridad, asegurando que la protección Constitucional se logre. Esta figura permite al Juzgador mantener las cosas en el estado en el que se encuentran, mediante la vía incidental; limitando con ello el actuar de las autoridades para garantizar la protección de los derechos humanos. ¿Qué pasa cuando el dejar de hacer de la Autoridad pone en peligro la materia del Amparo? En ese sentido, se documenta el caso de un hombre de la tercera edad que vive en condiciones muy precarias de pobreza y marginación a quien le fue concedida una suspensión a contrario sensu para que la autoridad responsable le proporcionara los servicios médicos necesarios. Se describen los supuestos en los que el Juez de Amparo dicta medidas de conservación y ordena a las autoridades continuar con el ejercicio de sus facultades jurisdiccionales con el fin de lograr la protección más amplia de los derechos humanos.

Palabras clave: Acto reclamado; constitucionalidad del acto reclamado; interés social; materia de amparo; protección; suspensión.

Cómo citar:

Rosiles-Sánchez, José Refugio. (2021). La suspensión del acto reclamado a "contrario sensu" del juicio de amparo. Revista de Investigaciones Universidad del Quindio, 33(S2), 39-48. https://doi.org/10.33975/riuq.vol33nS2.609 


\begin{abstract}
This document sets out the suspension of the act claimed as the Procedural Institution that it is, its importance and significance, whose purpose is to keep alive the matter that gives rise to the Amparo Trial, freezing the actions of the Authority, ensuring that Constitutional protection is achieved. This figure allows the Judge to keep things in the state in which they are, by incidental means; thereby limiting the actions of the authorities to guarantee the protection of human rights. What happens when the failure of the Authority endangers the matter of the Amparo? In this sense, the case of an elderly man living in very precarious conditions of poverty and marginalization is documented, who was granted a suspension a Contrary Sensu so that the responsible authority could provide him with the necessary medical services. The cases in which the Amparo Judge dictates conservation measures and orders the authorities to continue with the exercise of their jurisdictional powers in order to achieve the broadest protection of human rights are described.
\end{abstract}

Keywords: Claimed act; constitutionality of the claimed act; protection; protection matter; social interest; suspension.

\title{
INTRODUCCIÓN
}

Desde la década de los cincuenta del siglo XX, Couto (1973) sostuvo la necesidad de otorgar a la suspensión los efectos de un amparo provisional, para lo cual era necesario realizar un análisis previo de la probable inconstitucionalidad del acto reclamado la fracción X del artículo 107 de la Carta Magna-CPEUM que dispone que debe prever la naturaleza de la violación alegada.

Conforme a la anterior ley de amparo, en caso de incumplimiento por parte del responsable del auto que concedió la suspensión, lo precedente era la denuncia de violación de la medida cautelar en la vía incidental, conforme a lo previsto por los artículos 358 y 360 del Código Federal de Procedimientos Civiles. (Martínez, Hugo. 2015)

En este supuesto, la suspensión en el juicio de amparo indirecto era concedida analizando el interés con el que comparecía el quejoso, siempre tomando en cuenta la naturaleza la violación alegada, sin originar perjuicio de interés social ni se contravengan disposiciones de orden público (Tesis: PC.IV.C. J/8 C (10a.), 2017)

Después de la reforma al artículo primero de la CPEUM (Congreso de la Unión, art. 1, 9 de junio del 2012) el Estado se encuentra sujeto y pendiente de la estricta observancia de los derechos humanos reconocidos y contenidos en el mismo cuerpo normativo y en los Tratados Internacionales.

Esta reforma impactó directamente los juicios de tutela constitucional (juicio de amparo) y en particular a la protección que estos brindan a los gobernados a través de la institución procesal denominada suspensión del acto reclamado.

La investigación resulta oportuna al analizar el caso de la suspensión del acto reclamado concedida en los autos del juicio de amparo indirecto número: 452/2016-III, del índice del Juzgado Tercero del Décimo Circuito en el estado de Tabasco, en el que el quejoso, una persona de la tercera edad, en condiciones de pobreza, sin familia, enfermo y con una atención médica que se catalogó deficiente o casi nula, demandó al Estado la protección de su derecho humano a la salud, a un trato humano y digno. 
Con la suspensión concedida se logra mantener viva la materia que da origen al juicio de amparo, en este caso la vida del propio quejoso, asegurando con esto la protección constitucional de sus derechos humanos, pero con una particularidad, la suspensión se concedió a Contrario Sensu, lo que se traduce en un no dejar de hacer como legalmente establece la Ley de Amparo, Reglamentaria de los artículos 103 y 107 de la CPEUM, al respecto Mario Flores (2009) acertadamente señala:

“...la suspensión del acto reclamado como aquella medida cautelar que proveerá de vida al propio juicio, con una importancia que puede llegar a ser mayor, incluso, que el dictado de la sentencia protectora misma."

De lo anterior, se confirma que lo esencial en la suspensión es mantener viva la materia del juicio de amparo, pues sin ella, el juzgador no tendría elemento sobre el cual dictar una resolución (Gerónimo et al., 2021), trayendo consigo un desgaste innecesario en el sobrecargado sistema judicial.

No es óbice mencionar que concedida la suspensión se fija garantía para responder por los daños y perjuicios que la promoción de un juicio con la sola intención de retrasar u obtener un beneficio indebido frente a un tercero, posterior a ello se procede a fijar la contragarantía para levantar la suspensión, aunque previamente no se haya fijado garantía para obtenerla. (Tesis: VI.3o.A.10 K (10a.), 2017) En la especie la garantía señalada no aconteció por tratarse de una violación a un derecho fundamental y por no existir tercero extraño a juicio que pudiera alegar el perjuicio que se menciona en el párrafo inmediato anterior.

Analizado lo anterior, surge la pregunta: ¿Qué pasa cuando el dejar de hacer de la Autoridad pone en peligro la materia del Amparo? Para abordar el resultado de este cuestionamiento, resulta necesario acotar que este planteamiento llevará a entender los supuestos en los que el Juez de Amparo dicta medidas de conservación y ordena a las autoridades continuar con el ejercicio de sus facultades jurisdiccionales con el fin de lograr la protección más amplia de los derechos constitucionales violados, aspectos que se abordarán a continuación.

\section{METODOLOGÍA}

Para esta investigación se procedió a realizar una consulta de material bibliohemerográfico, fue basado en una metodología cualitativa con alcance descriptivo, analítico e interpretativo, empleando el método exegético.

\section{RESULTADOS}

\section{Aspectos básicos que llevaron a la tutela jurisdiccional efectiva.}

Después de la reforma constitucional de antes referida, nace una nueva forma de suspender los actos de las autoridades señaladas como responsables, pues adiciona la cultura de los derechos humanos a la CPEUM y por ende a la comunidad jurídica nacional, facultando a los jueces de distrito a ponderar de forma eficiente la máxima protección de esos derechos y de la dignidad humana frente al actuar de las autoridades, pues esto constituye el núcleo básico de protección del Estado, como lo señala la Suprema Corte de Justicia de la Nación (SCJN) al resolver el amparo en revisión 811/2008.

El caso de análisis es el juicio de amparo indirecto número 452/2016-III radicado ante el Juzgado Tercero del Décimo circuito en el estado de Tabasco. 
Para generar un antecedente se realiza una narrativa abreviada de hechos de la demanda de referencia, de lo que se desprende lo siguiente:

- Que se trata de una persona de la tercera edad que vive en condiciones muy precarias de pobreza y marginación.

- Durante su adolescencia y su vida adulta la única actividad laboral que desempeñó y con la cual sobrevivió fue el boxeo profesional.

- Nació en el año de 1948. Cuenta con estudios hasta el segundo año de primaria después de eso se dedicó a trabajar como ayudante en un gimnasio de Box. Este trabajo le permitió entrenar y desarrollarse con este deporte, llegando a participar en peleas profesionales, siendo su última pelea profesional en el año de 1973.

- Debido a la profesión elegida se le generaron problemas de salud cómo lo son: una fuerte anemia y daño en los riñones, situación que lo ha tenido interno en hospitales en diversas ocasiones.

- Siendo la última ocasión hospitalizado en el mes de diciembre del año 2015 en el hospital regional de alta especialidad donde lo estabilizaron y le diagnosticaron que estaba muy mal de los riñones, de la próstata y que cursaba un problema bastante agudo de diabetes.

- Esta enfermedad lo orilló a sortear cómo pudo los gastos de hospital que incluía las medicinas, algunos estudios y su manutención.

- Al ser una persona afiliada al seguro popular, seguro que acoge servicios de medicamentos, servicios y medicamentos no suministrados oportunamente por el hospital regional de alta especialidad.

- Fue en este mismo hospital donde le prescribieron el procedimiento de hemodiálisis dos veces por semana, sin embargo, la regularidad y la calidad de este servicio no le fue brindado como le había sido prescrito. Además, al asistir a las citas programadas lo hacían esperar hasta 19 horas en la sala de espera del hospital para ser atendido.

Cabe hacer énfasis en que el quejoso al referir los derechos humanos violado en su perjuicio enuncia en su demanda de garantías el artículo 4 de la CPEUM que contiene el derecho humano a la salud, y aduce que por ello tiene derecho a recibir atención médica profesional, oportuna y adecuada al grave padecimiento que sufre y que fueron prescritos por el propio hospital regional de alta especialidad, pues este artículo no solo menciona la garantía de acceso, sino también señala la carga del Estado para definir adecuadamente las bases para garantizar el acceso a los servicios de salud, artículo que en el párrafo IV dice:

“Toda persona tiene derecho a la protección de la salud. La Ley definirá las bases y modalidades para el acceso a los servicios de salud y establecerá la concurrencia de la Federación y las entidades federativas en materia de salubridad general, conforme a lo que dispone la fracción XVI del artículo 73 de esta Constitución. La Ley definirá un sistema de salud para el bienestar, con el fin de garantizar la extensión progresiva, cuantitativa y cualitativa de los servicios de salud para la atención integral y gratuita de las personas que no cuenten con seguridad social.” (2019)

Bajo esa tesitura, el quejoso promovió su demanda de amparo y solicitó: “...le fueran proporcionados los servicios médicos, quirúrgicos, farmacéuticos y hospitalarios de manera efectiva, oportuna, sin erogar más gastos, sin discriminación.” (León, 2016)

El derecho a la salud es un derecho humano, cuya prerrogativa es inherente a las personas. La citada reforma a la CPEUM (2011) a la luz de los tratados internacionales, establece los principios que se deben respetar para el reconocimiento expreso de los derechos humanos. 
Estos principios son:

Universalidad, por ser derechos inalienables de todos los seres humanos sin distinción alguna de persona por ningún motivo de raza, edad, género, religión, salud, preferencias, ideas, condición física, económica, social y cultural.

Interdependencia, la afectación o satisfacción de algún derecho llega a impactar otros u otros derechos.

Indivisibilidad, no se puede distinguir algún orden de importancia ya que se encuentran interrelacionados, no existe preeminencia entre los derechos otorgados.

Progresividad, en una sociedad de constante y evolutiva transformación de la dinámica social estos derechos pueden llegar a ser expansivos de acuerdo con nuevas condiciones sociales que determinen la vigencia y necesidad de otras prerrogativas que deban ser reconocidas de forma favorable al sujeto. Es la pobreza la que le impide tener acceso a servicios médicos particulares, teniendo como única opción la atención médica en el hospital regional de alta especialidad.

CONEVAL (2010), señala "que una persona es pobre cuando no tiene garantizado el ejercicio de al menos uno de sus derechos (derechos sociales) y si sus ingresos son insuficientes para adquirir los bienes y servicios que requiere para satisfacer sus necesidades básica”.

Héctor Fix Zamudio opina que:

"Es indudable que la suspensión de los actos reclamados constituye una providencia cautelar, por cuanto que significa una apreciación preliminar de la existencia de un derecho con el objeto de anticipar provisionalmente algunos efectos de la protección definitiva y por este motivo, no solo tiene eficacia puramente conservativa, sino que también puede asumir el carácter de una providencia constitutiva o parcial y provisionalmente restitutoria, cuando tales efectos sean necesarios para conservar la materia del litigio o impedir perjuicios irreparables o los interesados". (Del Arenal, 2015)

Silva (2014) por su parte señala que para hacer posible una cultura de convivencia remite a ubicar el conflicto en el contexto determinado, reconociendo sus elementos, propiedades y características para su prevención, atención y posible solución. Además de considerar los elementos de diálogo, comunicación y escucha activa (Silva, 2020).

Una vez que identificamos y se analiza la norma jurídica sujeta a interpretación y se descubre que "...en todo caso expresan la implicación de un consecuente por un antecedente" (García, 1955), implicando que la revisión de los antecedentes es de suma importancia, pero todo tiende a interpretación y para ello la búsqueda de tesis con resoluciones similares fortalecen los casos.

De ahí toma relevancia el análisis que se plantea, pues genera, si bien no un antecedente reconocido por la SCJN, si un criterio adoptado por los Juzgado de Distrito y que llevó a conceder la medida que se estudia, en la época en que se dictó. 


\section{Ley de Amparo, Reglamentaria de los artículos 103 y 107 de la Constitución Política de los Estados Unidos Mexicanos}

La suspensión se encuentra contenida en el art. 125 de la Ley de Amparo y establece que la suspensión del acto reclamado en el amparo indirecto documentado se puede decretar de oficio o de a petición del quejoso.

Si bien, la medida fue dictada bajo petición de parte agraviada, existe la doctrina que dice que "la suspensión de oficio tiende a la protección de los derechos personalísimos del agraviado, en todos los casos en los que se ataque su condición de hombre, y por excepción opera la medida de oficio en el aspecto patrimonial cuando se trata de proteger un valor insustituible que no puede restituirse si llegare a ser destruido, ni resarcirse por ser una calidad inherente a la cosa y que tampoco es apreciable en dinero" (Soto \& Liebana, 1977) supuesto que, si bien operaba, en la especie no sucedió.

La suspensión fue solicitada por el quejoso mediante incidente de suspensión, es decir, a petición de parte a través de una interlocutoria que en un primer momento concedió la provisional y posteriormente otorgó la definitiva dice los siguiente:

"En la ciudad de Villahermosa, Tabasco siendo las 09:08 del 31 de marzo de 2016 día y hora señalados por este juzgado de distrito para que tenga verificativo la presente audiencia incidental... Abierto el período probatorio... Abierto el período de alegatos... Resultandos... Considerandos quinto. Concesión de la suspensión definitiva: Toda vez que está en entredicho el derecho a la salud del quejoso JUAN ARTURO LEÓN HERNÁNDEZ, que de no concederse y si se consuma podría ser imposible restituir al quejoso de su derecho violado, por lo que procede conceder la suspensión provisional solicitada para que se le brinde atención médica al quejoso tendiente a atender su padecimiento, por lo que debe estar bajo la supervisión de los doctores especializados en el centro hospitalario que señala como autoridad responsable (Doctor Juan Graham Casasús); así como proporcionarle los medicamentos adecuados. Asimismo, se requiere a las autoridades responsables a fin de que informen periódicamente la evolución clínica del mencionado quejoso, a fin de estar en aptitud de proveer lo conducente en caso de su recuperación; con el apercibimiento de que de no hacerlo así; se le impondrá una multa de... Segundo. - se concede a JUAN ARTURO LEÓN HERNÁNDEZ la suspensión definitiva del acto reclamado a las autoridades responsables; en términos del considerando quinto de esta interlocutoria.” (León, 2016)

Para seguir profundizando en el tema, se abordan los supuestos planteados por la Primera Sala de la Suprema Corte de Justicia de la Nación al resolver la Contradicción de tesis 85/2018. El 16 de enero de 2019, dio origen a la tesis jurisprudencial con carácter de vinculante con número de registro: 2021263, misma que al rubro dice: "Suspensión. La naturaleza omisiva del acto reclamado no impide su procedencia” (EUM. SCJN. Primera Sala, 2019)

Criterio jurisprudencial que se generó dos años después de dictada la medida, pero que, a manera de criterio por los Jueces de Distrito, ya venía operando.

El máximo Tribunal del País, al analizar la fracción X, primer párrafo del Artículo 107 Constitucional y el Artículo 147 de la Ley de Amparo vigente, señalan que estos dotan a la suspensión de un genuino carácter de medida cautelar, haciendo referencia entonces que no solamente se trata de mantener las cosas en el estado en el que se encuentran, sino, van más allá al arribar a la premisa que su fin 
consiste en conservar la materia de la controversia, es decir, se dicta sentencia que falle sobre la constitucionalidad o no de los actos reclamados a la autoridad o autoridades responsables.

Continúa analizando que entonces el Juez, en pleno ejercicio de su actividad jurisdiccional debe dictar medidas conservativas o de tutela anticipada (efectos restitutorios), que permitan preservar la materia de la controversia. Pero siempre filtrando su razonamiento a través de los principios de la apariencia del buen derecho; las posibles afectaciones al interés social; $y$, la posibilidad jurídica y material de otorgar la medida.

Por ello, al analizar la naturaleza del acto reclamado, el juez debe arribar a la conclusión si estos tienen una actitud positiva, declarativa o negativa, y con base a esto, conceder o negar la suspensión solicitada, ponderando siempre las consecuencias de los efectos que esta pueda producir en el gobernado, aún y cuando impliquen restituir provisionalmente al quejoso en el goce del derecho violado. Lo que sucedió en la especie.

Concluyendo entonces que cuando la autoridad deja de hacer lo que por mandato expreso de la norma que la faculta y autoriza, deberán entenderse estos como actos de naturaleza omisiva y que llegar a esta deducción impacta positivamente en la determinación de la suspensión, al garantizar así que la persona que pretende la restitución de los derechos fundamentales dañados alcance, al menos momentáneamente el beneficio que pretende y que este pueda quedar firme al concluir el juicio.

Todo lo anterior, deberá desagregarse de la procedencia o no de la medida debido a su justificación al momento de solicitarla, es decir, demostrar al menos presuntivamente que es verdad y acreditar la legitimación del derecho que se pretende obtener.

Esta justificación fue posible de demostrar, con los informes previos rendidos por las autoridades responsables, aunque es de precisar que, en la práctica de la abogacía, en caso de negarse los actos, se convierte la carga de la prueba al quejoso y se debe estar muy atento para no perder la oportunidad de demostrar el acto con las pruebas que la ley de amparo permite en el incidente de suspensión. (Art. 143, segundo párrafo)

\section{Importancia del acceso a la salud como derecho humano y su oportuna protección en el Incidente de Suspensión del Juicio de Amparo.}

En el caso que nos ocupa la autoridad jurisdiccional, denominado juez de distrito, después de ponderar bajo los principios de la apariencia del buen derecho y la nueva afectación social llegó a la conclusión de que el derecho vertido en la demanda de amparo era el derecho a la salud, y por consecuencia en estricta observancia del artículo primero de la CPEUM, dictará una medida que satisficiera el respeto irrestricto al derecho humano violado, derecho a la salud contenido en el artículo cuarto párrafo cuarto de la CPEUM e instrumentos internacionales en materia de derechos económicos, sociales y culturales; así como lo relativo a la corte interamericana de derechos humanos en materia de hechos económicos sociales y culturales.

Para hacer un mayor énfasis resulta necesario transcribir el artículo cuarto, párrafo cuarto, de la Constitución General de la República que dice lo siguiente:

“Toda persona tiene derecho a la protección de la salud. La ley definirá las bases y modalidades para el acceso a los servicios de salud y establecerá la concurrencia de la Federación y de las 
entidades federativas en materia de salubridad general, conforme lo dispone la fracción $16^{\mathrm{a}}$ del artículo 73 de esta Constitución."

Fue después de esta interpretación conforme y literal (Caballero, 2019) de la norma que el juez ponderó el derecho a la salud del quejoso, haciendo énfasis en que constituye un derecho fundamental que de no tutelar se, y de consumarse el acto reclamado, podría ser imposible restituirlo, por lo que concede la suspensión provisional para que se le brinde atención médica al quejoso para su padecimiento, debiendo estar en supervisión de médicos especialistas en el centro hospitalario que señala como autoridad responsable y que deberá proporcionarle los medicamentos adecuados para su padecimiento. Debiendo la autoridad responsable informar sobre el cumplimiento de esta medida en el término de 24 horas.

Posterior a esto y habiendo cumplido con las formalidades que ley de amparo señala para estar en condiciones de resolver en definitiva el incidente de suspensión el mismo juez de distrito dictó resolución interlocutores en la que concedió la suspensión definitiva en los siguientes términos:

"Toda vez que está en entredicho el derecho a la salud del quejoso, lo que constituye un derecho fundamental que no concederse y si se consumó podría ser imposible restituir Al quejoso de su derecho violado, por lo que procede conceder la suspensión provisional solicitada para que se le brinda atención médica al quejoso tendiente a atender padecimiento, por lo que debe estar bajo la supervisión de los doctores especializados en el centro hospitalario que señala como autoridad responsable; así como proporcionar los medicamentos adecuados. Asimismo, se requiere a las autoridades responsables a fin de que informen periódicamente la evolución clínica del mencionado quejoso, a fin de estar en aptitud de proveer lo conducente en caso de su recuperación; cuál es el apercibimiento que de no hacerlo así; se le impondrá una multa de... Segundo. Se concede al quejoso la suspensión definitiva del acto reclamado las autoridades responsables en términos del considerando quinto este interlocutoria."

\section{DISCUSIÓN}

Citando a Juventino V. Castro distingue que "la suspensión del acto reclamado en el proceso de amparo, es un proveído dictado incidentalmente que tiene por objeto y como función preservar la materia del juicio, mediante la paralización transitoria de los efectos jurídicos y materiales del acto de autoridad cuya constitucionalidad se controvierte, mientras se dicta la sentencia definitiva en el proceso, o se sobresee éste por la improcedencia declarada de la acción” (Castro, 1979).

La naturaleza jurídica de la suspensión pudiera ser provisional por lo que radica en que es una medida transitoria que opera en forma temporalmente limitada, pues solo dura unos días, mientras se resuelve sobre la suspensión definitiva de los actos reclamados. (Tesis: Semanario Judicial de la Federación)

La suspensión definitiva se distingue de la provisional debido al mandamiento por medio del cual se decreta, así como en el tiempo de su duración, ya que la provisional se decreta en el auto que abre el incidente de suspensión, el cual surte efectos, hasta en tanto no se dicte la definitiva y ésta se resuelve en una resolución interlocutoria, que tiene vigencia hasta que se notifica la sentencia ejecutoriada de amparo. (Ojeda, 2009).

La suspensión decretada en el Juicio de amparo, su protección y garantía, tuvo a bien mantener con vida al quejoso frente a los actos omisivos de la autoridad, al negarse a proporcionarle la atención 
médica adecuada a su padecimiento y con ello satisfacer el interés del quejoso en recibir el tratamiento que por derecho humano debe tener acceso.

La característica especial de la suspensión documentada es que no se trató en un dejar de hacer como la norma lo señala, sino, en continuar haciendo para lograr la garantía proteccionista que el juicio pregona. La suspensión a contrario sensu permitió que lejos de un dejar de hacer de la autoridad señalada como responsable, ésta fuera obligada a prestar de forma eficiente el servicio para el cual fue constituida, salvaguardando así la vida del quejoso, su derecho humano a la salud y recibiendo el trato digno que merece, no solo por su condición de persona, sino, por su avanzada edad.

\section{REFERENCIAS}

1. Caballero Ochoa, José Luis. (2019). La interpretación conforme en el escenario jurídico mexicano. Algunas pautas para su aplicación a cinco años de la reforma constitucional de 2011. Revista CEC. Recuperado de: https:/www.sitios.scjn.gob.mx/cec/sites/default/files/publication/ documents/2019-03/06 CABALLERO_REVISTA\%20CEC 03.pdf

2. CONEVAL. (2010). Evolución de los determinantes de la pobreza de las principales ciudades de México 1990-2010. México. Consejo Nacional de Evaluación de la Política de Desarrollo Social.

3. Couto, Ricardo. (1973). Tratado teórico práctico de la suspensión en el amparo. Porrúa.

4. Del Arenal Martínez, Vicente Roberto. (2015) La técnica en el juicio de amparo. Editorial Flores. p. 574.

5. Estados Unidos Mexicanos. Congreso Constituyente. (1917). Constitución Política de los Estados Unidos Mexicanos. [Ley Fundamental]. Diario Oficial de la Federación: 05-02-1917. Recuperado de http://www.diputados.gob.mx/LeyesBiblio/ref/cpeum.htm

6. Estados Unidos Mexicanos. Congreso General. (29 de diciembre de 2014). Ley de Amparo, Reglamentaria de los artículos 103 y 107 de la Constitución Política de los Estados Unidos Mexicanos. DOF: 15-06-2018. Recuperado de: http://www.diputados.gob.mx/LeyesBiblio/pdf/ LAmp_150618.pdf

7. Estados Unidos Mexicanos. Suprema Corte de Justicia de la Nación. Primera Sala. (2019) SUSPENSIÓN. LA NATURALEZA OMISIVA DEL ACTO RECLAMADO NO IMPIDE SU PROCEDENCIA. [Tesis Jurisprudencial 1a./J. 70/2019 (10a.)] Gaceta del Semanario Judicial de la Federación. Libro 73, diciembre de 2019, Tomo I, (p. 286).

8. Flores, Mario. (2009). La medida cautelar en el proceso constitucional del amparo; necesidad de replantear su tradicional interpretación. Poder Judicial de la Federación. Recuperado de: https:// www.supremacorte.gob.mx/sites/default/files/transparencia/documentos/becarios/019mariocesar-flores-munoz.pdf

9. García Máynez, Eduardo. (1955) Lógica del juicio jurídico, Diánoia-revista de filosofía-, UNAMFCE, vol. I, núm. 1, pp. 3-23.

10. Gerónimo González, Paulino, Silva Hernández, Francisca \& Martínez Prats, Germán. (2021). Mecanismos alternativos en la solución de conflictos para la construcción de una cultura de paz. Revista ciencias de la documentación, 7(1), 15-23.

11. Juan Arturo León Hernández Vs C. Gobernador Constitucional del Estado de Tabasco, entre otros, Exp. 452/2016-III (Juzgado Tercero de Distrito del Décimo Circuito en el estado de Tabasco)

12. Martínez García, Hugo (2015). La suspensión del acto reclamado en la nueva ley de amparo. Rechtikal, p. 370.

13. Ojeda Bohórquez, Ricardo. (2009). Teoría de la suspensión del acto reclamado en materia penal, Editorial Porrúa, (pp.93-94)

14. Silva Hernández, Francisca. (2014) Mediación comunitaria como mecanismo alternativo para el tratamiento de conflictos en pueblos indígenas. Mediación y Derechos Humanos. Porrúa, México.

15. Silva-Hernández, Francisca. (2020). Hacia una comprensión del conflicto en justicia alternativa. Revista de Investigaciones Universidad del Quindío, 32(1), 61-65. https://doi.org/10.33975/riuq. vol32n 1.435

16. Soto, Ignacio y Liebana, Gilberto. (1977). La suspensión del acto reclamado en el juicio de amparo. 
Porrúa. Pp.53-54.

17. Tesis: VI.3o.A.10 K (10a.). Gaceta del Semanario Judicial de la Federación, Décima Época, 2014740, Tribunales Colegiados de Circuito Libro 44, Julio de 2017, Tomo II, Pág. 1098, Tesis Aislada (Común)

18. Tesis: Semanario Judicial de la Federación. Quinta Época, 343600. Tercera Sala de la Suprema Corte de Justicia de la Nación. t. CV, p. 1030 Tesis Aislada (Común)

19. Tesis: PC.IV.C. J/8 C (10a.). Gaceta del Semanario Judicial de la Federación, Décima Época, 2015557, Plenos de Circuito, Libro 48, noviembre de 2017, Tomo II, Pág. 1573, Jurisprudencia.

20. Castro y Castro, Juventino V. (1979) El sistema de derecho de amparo. Editorial Porrúa, p.175. 